\title{
Methoden
}

Saman Khodaverdian* und Jürgen Adamy

\section{Entkopplungsbasierte Synchronisierung heterogener linearer Multi-Agenten-Systeme}

\author{
Decoupling-based Synchronization of Heterogeneous Linear Multi-Agent Systems
}

Zusammenfassung: Der vorliegende Beitrag befasst sich mit der Synchronisierung heterogener linearer Systeme. Es wird eine dezentrale Regelungsstrategie vorgestellt, mit deren Hilfe die Ausgangsdynamik der nicht identischen Systeme homogenisiert und gezielt vorgegeben wird. Anschließend lassen sich bekannte Resultate für die Synchronisierung homogener Systeme anwenden. Hierauf aufbauend wird noch eine Erweiterung präsentiert, die die exakte Vorgabe einer Wunschtrajektorie als Synchronisierungstrajektorie erlaubt.

Schlüsselwörter: Synchronisierung, dezentrale Regelung, Multi-Agenten-Systeme, heterogene Netzwerke.

\begin{abstract}
This paper deals with the synchronization of heterogeneous linear systems. A decentralized control strategy is presented to homogenize the output dynamics of the systems selectively. Then, the results for synchronizing homogeneous systems can be applied. Based on this, an extension is presented which allows to specify the synchronization trajectory explicitly.
\end{abstract}

Keywords: Synchronization, decentralized control, multiagent systems, heterogeneous networks.

DOI 10.1515/auto-2014-1109

Eingang 2. April 2014; angenommen 23. Juli 2014

\section{Einleitung}

Die kooperative Regelung von vernetzten dynamischen Systemen bzw. Multi-Agenten-Systemen ist ein in jüngster Zeit intensiv erforschtes Themengebiet. Mit Hilfe von Agenten-Netzwerken lassen sich diverse Aufgaben effizient lösen. Als mögliche Anwendungsbeispiele können un-

*Korrespondenzautor: Saman Khodaverdian, Technische Universität Darmstadt, E-Mail: saman.khodaverdian@rmr.tu-darmstadt.de Jürgen Adamy: Technische Universität Darmstadt ter anderem die Multi-Roboter-Exploration, die Formationsregelung, die Handhabung von Gefahrengüter oder die kollektive Überwachung sicherheitskritischer Anwendungen genannt werden. Die Artikel [19] und [21] geben eine umfassende Übersicht zu den Einsatzmöglichkeiten von Multi-Agenten-Systemen und fassen aktuelle Forschungsergebnisse zusammen.

Eine grundlegende Zielsetzung zur Erfüllung solcher Aufgaben besteht in der Synchronisierung der Agenten, also in der Erreichung eines gemeinsamen Zustands. Dabei wird i.d.R. eine dezentrale Lösung angestrebt, d.h., die Synchronisierung soll ohne eine übergeordnete zentrale Instanz erreicht werden. Dies hat den Vorteil, dass Agentenausfälle kompensiert werden können. Zu diesem Zweck tauschen die Agenten über ein Kommunikationsnetzwerk Informationen untereinander aus, die in einer dezentralen Reglerstruktur verarbeitet werden, um eine Zustandssynchronisierung zu erreichen.

Innerhalb der letzten Jahre wurden viele Ergebnisse für die Synchronisierung homogener, also identischer, Agenten erzielt. Während anfänglich Systeme mit reiner Integratordynamik $[20,21]$ betrachtet wurden, hat sich der Forschungsschwerpunkt mittlerweile auf Systeme mit allgemeiner linearer Dynamik [5, 14, 18, 25] verlagert. Aufbauend auf diesen Resultaten ist die Analyse von heterogenen, also nicht identischen, Agenten ein aktuell forschungsintensives Gebiet. Aus praktischen Gesichtspunkten ist die Betrachtung von Agenten mit individueller Dynamik besonders interessant, da durch den Einsatz von heterogenen Multi-Agenten-Systemen eine neue Bandbreite von Einsatzmöglichkeiten eröffnet wird.

In einem Netzwerk von heterogenen Agenten besteht das Ziel darin, eine bestimmte Untermenge der Agentenzustände zu synchronisieren. In der Literatur ist das unter dem Problem der Ausgangssynchronisierung bekannt. Da heterogene Agenten unterschiedliche Zustandsdimensionen, mit möglicherweise unterschiedlichen physikalischen Bedeutungen, aufweisen können, ist eine vollständige Zustandssynchronisierung i.d.R. nicht möglich oder sinnvoll. Es ist bekannt, dass die Agenten zur Lösung die- 
ses Problems ein sogenanntes Inneres-Modell-Prinzip erfüllen müssen [11, 17, 27]. Demnach muss jeder Agent die Dynamik der Synchronisierungstrajektorie als inneres Modell enthalten.

Bislang existieren nur wenige Ergebnisse zur Lösung dieses Problems. In der Literatur haben sich jedoch bereits zwei verschiedene Ansätze entwickelt. Im ersten Ansatz wird vorausgesetzt, dass ausschließlich relative Informationen zur Verfügung stehen. D.h., die Agenten haben keinerlei Kenntnis über ihre internen Zustände und können nur relative Zustandsdifferenzen zu ihren Nachbarn messen $[10,15]$. Dies ist beispielsweise der Fall, wenn Agenten innerhalb eines unbekannten Gebiets ihre absolute Position nicht kennen, aber ihren Abstand $\mathrm{zu}$ benachbarten Agenten messen können.

Im zweiten Lösungsansatz wird angenommen, dass jeder Agent zumindest einen Teil seiner internen Zustände kennt bzw. messen kann. Diese Informationen können dann für eine vorgelagerte Regelung genutzt werden, um die Agenten zu homogenisieren und die bekannten Resultate für die Synchronisierung identischer Agenten $\mathrm{zu}$ verwenden. Eines der bislang wenigen Resultate hierzu wird in [29] präsentiert. Dabei werden mehrere dynamische Vorfilter genutzt, um ein bestimmtes Systemverhalten zu erzeugen. Neben einer hohen Dynamikordnung erfordert die Lösung jedoch eine spezielle Darstellungsform, wodurch der Entwurf sehr aufwändig und intransparent werden kann.

Der vorliegende Beitrag gliedert sich zum zweiten Lösungsansatz ein. Es wird eine zu [29] alternative Methode vorgestellt. Hierbei wird auf das Entwurfsverfahren der Entkopplungsregelung zurückgegriffen, die es erlaubt, die Ausgangsgrößen eines Agenten unabhängig voneinander zu beeinflussen. Der Entwurf ist vergleichsweise transparent und die Dynamikordnung des Regelgesetzes ist niedrig. Unter gewissen Voraussetzungen ist es sogar möglich mit einer rein statischen Zustandsregelung eine Ausgangssynchronisierung zu erhalten.

Die nachfolgenden Ausführungen gliedern sich wie folgt. Zunächst werden einige mathematische Grundlagen wiederholt und die Problemstellung wird definiert. In Abschnitt 2.4 werden außerdem bekannte Resultate zur Synchronisierung homogener Agenten zusammengefasst. Abschnitt 3 präsentiert einen auf den Entkopplungsreglerentwurf basierenden Homogenisierungsansatz, mit dessen Hilfe die Synchronisierung der heterogenen Agenten durchgeführt wird. Zusätzlich wird in Abschnitt 4 eine Möglichkeit aufgezeigt, die es erlaubt die Agenten auf eine vorgebbare Wunschtrajektorie zu synchronisieren. Zur Veranschaulichung der Resultate wird in Abschnitt 5 die Ausgangssynchronisierung für ein Netzwerk von lineari- sierten Flugzeugmodellen betrachtet, bevor die Ergebnisse in Abschnitt 6 zusammengefasst werden.

\section{Grundlagen}

\subsection{Notation}

Die quadratische Einheitsmatrix der Dimension $n$ wird als $I_{n}$ geschrieben. Entsprechend werden die Nullmatrix und der Einsvektor durch $\mathbf{0}$ und $\mathbf{1}$ dargestellt, wobei die Dimensionierungen jeweils aus dem Kontext hervorgehen. Eine Blockdiagonalmatrix wird verkürzt als $\boldsymbol{M}=\operatorname{diag}\left(\boldsymbol{M}_{1}, \ldots, \boldsymbol{M}_{p}\right)$ geschrieben. Für eine Matrix $\boldsymbol{K} \in$ $\mathbb{R}^{m \times n}$ gilt mit $n>m$ und rang $(\boldsymbol{K})=m$, dass $\boldsymbol{K} \boldsymbol{K}^{+}=\boldsymbol{I}_{m}$, wobei $\boldsymbol{K}^{+} \in \mathbb{R}^{n \times m}$ eine Rechtsinverse von $\boldsymbol{K}$ ist. Ihre Transponierte wird durch $\boldsymbol{K}^{\top}$ repräsentiert. Des Weiteren bezeichnet $\boldsymbol{k}_{i}$ die $\boldsymbol{i}$-te Spalte und $\boldsymbol{k}_{j}^{\top}$ die $\boldsymbol{j}$-te Zeile von $\boldsymbol{K}$. Das Kroneckerprodukt wird durch das Symbol $\otimes$ dargestellt.

\subsection{Problemstellung}

Betrachtet wird ein Multi-Agenten-System bestehend aus $N$ Agenten, die jeweils durch ein lineares zeitinvariantes Modell

$$
\begin{aligned}
& \dot{x}_{i}=A_{i} x_{i}+B_{i} u_{i}, \\
& y_{i}=C_{i} x_{i},
\end{aligned}
$$

mit $i \in\{1, \ldots, N\}$, beschrieben werden. Hierbei bezeichnen die Vektoren $\boldsymbol{x}_{i} \in \mathbb{R}^{n_{i}}, \boldsymbol{u}_{i} \in \mathbb{R}^{m_{i}}$ und $\boldsymbol{y}_{i} \in \mathbb{R}^{p}$ den Zustands-, Stellgrößen- und Ausgangsvektor des $i$-ten Agenten. Für das System (1) wird abkürzend $\left(\boldsymbol{A}_{i}, \boldsymbol{B}_{i}, \boldsymbol{C}_{i}\right)$ geschrieben. Es wird angenommen, dass für alle Agenten rang $\left(\boldsymbol{B}_{i}\right)=m_{i} \geq p$ gilt, d.h., jeder Agent weist mindestens $p$ linear unabhängige Aktoren auf. $\mathrm{Zu}$ beachten ist, dass die Agenten unterschiedliche Zustands- und Eingangsdimensionen haben können, die Dimension des Ausgangsvektors ist jedoch für alle Agenten $p$. Andernfalls wäre eine Ausgangssynchronisierung nicht möglich. Darüber hinaus sollten die Ausgangsgrößen der Agenten sinnvollerweise physikalisch miteinander vergleichbar sein. Formal lässt sich folgendes Problem definieren:

Problem 1. Gegeben seien $N$ Agenten der Form (1). Finde ein dezentrales Regelgesetz $\boldsymbol{u}_{i}=\boldsymbol{f}_{i}\left(\boldsymbol{x}_{i}, \boldsymbol{\eta}_{i}\right)$, sodass

$$
\lim _{t \rightarrow \infty}\left\|\boldsymbol{y}_{i}(t)-\boldsymbol{y}_{j}(t)\right\|=\mathbf{0}, \quad \forall i, j \in\{1, \ldots, N\} .
$$

Hierbei bezeichnet $\boldsymbol{\eta}_{i}$ den neuen Stellvektor des Systems, welcher in Abschnitt 3 eingeführt wird. Um triviale Lösun- 
gen zu vermeiden, wird vorausgesetzt, dass sich die Agenten auf eine Trajektorie synchronisieren, die nicht asymptotisch stabil ist.

Die Lösung von Problem 1 beruht auf der Möglichkeit, dass die Agenten miteinander kommunizieren und Informationen austauschen können. Dieser Informationsaustausch wird meist mit Hilfe von Graphen modelliert.

\subsection{Graphentheorie}

Die Kommunikationstopologie in einem Multi-AgentenSystem lässt sich mit Hilfe der algebraischen Graphentheorie in einfacher Art und Weise darstellen. Nachfolgend werden einige Grundlagen beschrieben, die für die Modellierung und Analyse der Kommunikation zwischen den Agenten benötigt werden. Für einen tiefer gehenden Einblick in die Thematik wird auf [9] verwiesen.

Gerichtete Graphen $\mathcal{G}=\left(\mathcal{V}_{\mathcal{G}}, \mathcal{E}_{\mathcal{G}}\right)$ bestehen aus einer endlichen Menge von Knoten $\mathcal{V}_{\mathcal{G}}=\left\{v_{1}, \ldots, v_{N}\right\}$ und Kanten $\mathcal{E}_{\mathcal{G}} \subseteq \mathcal{V}_{\mathcal{G}} \times \mathcal{V}_{\mathcal{G}}$. Hierbei wird jeder Agent im MultiAgenten-System durch einen Knoten $v_{i} \in \mathcal{V}_{\mathrm{g}}$ repräsentiert und der Informationsfluss wird durch gerichtete Kanten $\left(v_{i}, v_{j}\right) \in \mathcal{E}_{\mathcal{G}}$ dargestellt. Demnach kann Agent $i$ Informationen an Agent $j$ senden, wenn die Kante $\left(v_{i}, v_{j}\right)$ existiert. In diesem Artikel werden schleifenfreie Graphen betrachtet, d.h. $\left(v_{i}, v_{i}\right) \notin \mathcal{E}_{\mathrm{g}}$ für alle $i \in\{1, \ldots, N\}$. Eine wichtige Eigenschaft gerichteter Graphen stellt ihr Zusammenhang dar.

Definition 1. Ein gerichteter Graph wird als zusammenhängend bezeichnet, wenn mindestens ein Knoten $v_{i} \in \mathcal{V}_{\mathcal{G}}$ existiert, der direkt oder indirekt alle anderen Knoten im Graphen, unter Zuhilfenahme der gegebenen Kantenmenge $\mathcal{E}_{g}$, erreichen kann. Knoten dieser Art werden auch als Wurzelknoten bezeichnet.

Ist ein gerichteter Graph zusammenhängend, sagt man auch, dass er einen gerichteten Spannbaum enthält [28]. Abbildung 1 zeigt beispielhaft einen zusammenhängenden gerichteten Graphen, mit den Wurzelknoten $v_{1}$ und $v_{3}$.

Eine wichtige Matrix zur Beschreibung von Graphen ist die sogenannte Adjazenzmatrix $\boldsymbol{A}_{\mathcal{G}}=\left[a_{\mathcal{G}_{i j}}\right] \in \mathbb{R}^{N \times N}$. In ihr werden die Kantenverbindungen des Graphen zusammengefasst, wobei $a_{\mathcal{G}_{i j}}=1$, wenn $\left(v_{i}, v_{j}\right) \in \mathcal{E}_{\mathcal{G}}$ und $a_{\mathcal{G}_{i j}}=0$, wenn $\left(v_{i}, v_{j}\right) \notin \mathcal{E}_{\mathcal{G}}$. Die Gradmatrix wird als $\boldsymbol{D}_{\mathcal{G}}=\operatorname{diag}\left(d_{\mathcal{G}_{1}}, \ldots, d_{\mathcal{G}_{N}}\right) \in \mathbb{R}^{N \times N}$ definiert. Ihre Elemente $d_{\mathcal{G}_{j}}=\sum_{i=1}^{N} a_{\mathcal{G}_{i j}}$ geben die Anzahl aller eingehenden Kanten in die Knoten $v_{j}$ an. Mit Hilfe der Adjazenzmatrix und der Gradmatrix ergibt sich die Laplacematrix, die eine der wichtigsten Matrizen im Zusammenhang von Synchroni-

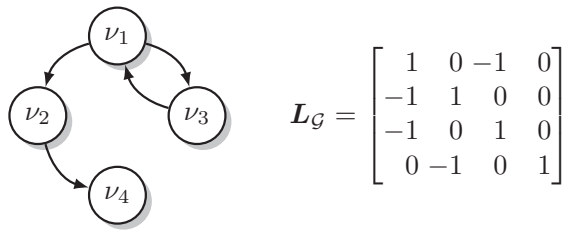

Abbildung 1: Beispiel für einen zusammenhängenden gerichteten Graphen mit zugehöriger Laplacematrix $\boldsymbol{L}_{\mathcal{G}}$. Die Knoten $v_{1}$ und $v_{3}$ stellen Wurzelknoten dar.

sierungsproblemen darstellt [19]. Sie berechnet sich aus $\boldsymbol{L}_{\mathcal{G}}=\boldsymbol{D}_{\mathcal{G}}-\boldsymbol{A}_{\mathcal{G}}^{\top}$. Über die Definitionsgleichung der Laplacematrix ist direkt ersichtlich, dass mindestens ein Eigenwert von $\boldsymbol{L}_{\mathcal{G}}$ bei 0 liegt, mit zugehörigem Eigenvektor 1 . Dies folgt aus der Tatsache, dass sich die Zeilensummen der Laplacematrix alle zu 0 ergeben, d.h. $\boldsymbol{L}_{\mathcal{G}} \mathbf{1}=\mathbf{0}$. Außerdem lässt sich zeigen, dass 0 genau dann ein einfacher Eigenwert ist, wenn der Graph zusammenhängend ist [13]. Eine weitere wichtige Eigenschaft über die Eigenwerte der Laplacematrix liefert der Satz von Gerschgorin, wonach alle von Null verschiedenen Eigenwerte von $\boldsymbol{L}_{\mathcal{G}}$ einen positiven Realteil aufweisen [12]. Zusammenfassend folgt für einen zusammenhängenden gerichteten Graphen, dass $\lambda_{1}\left(\boldsymbol{L}_{\mathcal{G}}\right)=0$ und $\mathfrak{R e}\left\{\lambda_{i}\left(\boldsymbol{L}_{\mathcal{G}}\right)\right\}>0$ für $i \in\{2, \ldots, N\}$.

\subsection{Synchronisierung homogener Agenten}

Die Lösung des Synchronisierungsproblems 1 beruht auf einem Homogenisierungsansatz. Aus diesem Grund werden in diesem Abschnitt einige Resultate zur Synchronisierung homogener Agenten zusammengefasst.

Gegeben sei ein Multi-Agenten-System bestehend aus den Agenten

$$
\dot{\boldsymbol{x}}_{i}=\boldsymbol{A} \boldsymbol{x}_{i}+\boldsymbol{B} \boldsymbol{u}_{i}, \quad i \in\{1, \ldots, N\},
$$

mit $\boldsymbol{x}_{i} \in \mathbb{R}^{n}, \boldsymbol{u}_{i} \in \mathbb{R}^{m}$ und den für alle Agenten identischen System- und Eingangsmatrizen $\boldsymbol{A}$ und $\boldsymbol{B}$. Unter der Voraussetzung, dass die Agenten Informationen über das Kommunikationsnetzwerk austauschen können, kann mit Hilfe des Regelgesetzes

$$
\boldsymbol{u}_{i}=-\boldsymbol{H} \sum_{j=1}^{N} a_{\mathcal{G}_{j i}}\left(\boldsymbol{x}_{i}-\boldsymbol{x}_{j}\right)
$$

und einer geeignet gewählten Matrix $H \in \mathbb{R}^{m \times n}$ eine Zustandssynchronisierung

$$
\lim _{t \rightarrow \infty}\left\|\boldsymbol{x}_{i}(t)-\boldsymbol{x}_{j}(t)\right\|=0, \quad \forall i, j \in\{1, \ldots, N\},
$$

erreicht werden. Fasst man die Agenten (3) mit ihren Regelgesetzen (4) zusammen und betrachtet den Gesamtre- 
gelkreis des Multi-Agenten-Systems, erhält man

$$
\dot{\boldsymbol{x}}=\left(\boldsymbol{I}_{n} \otimes \boldsymbol{A}-\boldsymbol{L}_{\mathcal{G}} \otimes \boldsymbol{B H}\right) \boldsymbol{x},
$$

wobei $\boldsymbol{x}^{\top}=\left[\boldsymbol{x}_{1}^{\top} \cdots \boldsymbol{x}_{N}^{\top}\right]$.

Satz 1. Für das Multi-Agenten-System (6) wird Synchronisierung gemäß (5) erreicht, wenn die Matrizen $\boldsymbol{A}$ $\lambda_{i}\left(\boldsymbol{L}_{\mathcal{G}}\right) \boldsymbol{B} \boldsymbol{H}$ für alle $i \in\{2, \ldots, N\}$ Hurwitz sind. In diesem Fall synchronisieren sich die Agenten auf eine Trajektorie, die durch

$$
\lim _{t \rightarrow \infty} \boldsymbol{x}_{i}(t)=e^{\boldsymbol{A} t}\left(\boldsymbol{r}_{\mathcal{G}}^{\top} \otimes \boldsymbol{I}_{n}\right) \boldsymbol{x}(0), \quad \forall i \in\{1, \ldots, N\},
$$

gegeben ist. Hierbei bezeichnet $\boldsymbol{r}_{\mathcal{G}}^{\top}$ den zugehörige Linkseigenvektor zum Eigenwert $\lambda_{1}\left(\boldsymbol{L}_{\mathcal{G}}\right)=0$.

Satz 1 stellt ein bekanntes Resultat für die Synchronisierung homogener Agenten dar, weshalb der Beweis nicht ausgeführt wird.

Aus (7) ist ersichtlich, dass bei Gültigkeit der Voraussetzungen von Satz 1 alle Agenten zu einer Trajektorie synchronisieren, deren Dynamik durch die Systemmatrix $\boldsymbol{A}$ festgelegt wird. Des Weiteren ist aus [28] bekannt, dass die Elemente von $\boldsymbol{r}_{\mathcal{G}}^{\top}$ positiv sind. Da $\mathbf{1}$ der Rechtseigenvektor zum Eigenwert $\lambda_{1}\left(\boldsymbol{L}_{\mathcal{G}}\right)=0$ ist, gilt außerdem $\boldsymbol{r}_{\mathcal{G}}^{\top} \mathbf{l}=1$. Demnach ergeben sich die Anfangswerte der Synchronisierungstrajektorie aus einem gewichteten Mittel der Anfangswerte aller Agenten, wobei die Gewichtungen durch den zu $\lambda_{1}\left(\boldsymbol{L}_{\mathcal{G}}\right)=0$ gehörigen Linkseigenvektor der Laplacematrix bestimmt sind. Dies verdeutlicht den Einfluss der Kommunikationsstruktur auf die Synchronisierungstrajektorie.

Damit sich eine Synchronisierung einstellen kann, muss also eine Matrix $\boldsymbol{H}$ gefunden werden, sodass gleichzeitig die Matrizen

$$
\boldsymbol{A}-\lambda_{i}\left(\boldsymbol{L}_{\mathcal{G}}\right) \boldsymbol{B H}, \quad \forall i \in\{2, \ldots, N\}
$$

Hurwitz sind. Dieses simultane Stabilisierungsproblem wurde in [25] mit Hilfe eines LQR-Ansatzes gelöst und wird in folgendem Lemma zusammengefasst.

Lemma 1. Angenommen die Kommunikation zwischen den Agenten (3) werde durch einen zusammenhängenden Graphen beschrieben und das Paar $(\boldsymbol{A}, \boldsymbol{B})$ sei stabilisierbar. Dann sind die Matrizen (8) Hurwitz, wenn $\boldsymbol{H}=\sigma \boldsymbol{B} \boldsymbol{P}$ gewählt wird. Hierbei ist $0<\sigma \leq \min _{i \geq 2}\left\{\mathfrak{R e}\left\{\lambda_{i}\left(\boldsymbol{L}_{\mathcal{G}}\right)\right\}\right\}$ und die symmetrische positiv definite Matrix $\boldsymbol{P} \in \mathbb{R}^{n \times n}$ berechnet sich aus der algebraischen Riccati-Gleichung

$$
\boldsymbol{P A}+\boldsymbol{A}^{\top} \boldsymbol{P}-\sigma \boldsymbol{P B B ^ { \top }} \boldsymbol{P}+I_{n}=\mathbf{0} .
$$

Für einen Beweis von Lemma 1 wird auf [25] verwiesen.

\section{Homogenisierung durch Entkopplung}

Im Folgenden wird nun eine Homogenisierungsmethode vorgestellt, um die Resultate aus dem vorangegangenen Abschnitt auf die heterogenen Agenten (1) anwendbar zu machen.

\subsection{Entkopplungsregler}

Die Entkopplungsregelung ist ein intensiv erforschtes Gebiet in der Regelungstechnik. Die Idee besteht darin, durch eine Führungsentkopplung jede Ausgangsgröße gezielt durch eine ihr zugeordnete Führungsgröße zu beeinflussen und von der restlichen Systemdynamik zu entkoppeln. Dies hat den Vorteil, dass die Änderung einer Führungsgröße lediglich die zugehörige Ausgangsgröße beeinflusst. Dadurch lässt sich ein MIMO-System mit $p$ Ausgängen auf $p$ SISO-Systeme zurückführen, die jeweils einzeln analysiert und geregelt werden können. In diesem Artikel wird die Entkopplungsregelung nicht nur dazu genutzt eine Führungsentkopplung zu generieren, sondern auch um die einzelnen Agenten zu homogenisieren. Nachfolgend wird die Entwurfsmethodik nach [4] zur Entkopplung linearer MIMO-Systeme vorgestellt. Eine alternative Herangehensweise bietet der Entwurf nach der Vollständigen Modalen Synthese [22].

Das Ziel bei der Entkopplungsregelung besteht darin, einen Zustandsregler

$$
\boldsymbol{u}_{i}=-\boldsymbol{K}_{i} \boldsymbol{x}_{i}+\boldsymbol{F}_{i} \boldsymbol{\eta}_{i}
$$

so auszulegen, dass der geschlossene Regelkreis aus (1) und (9) eine diagonale Übertragungsmatrix aufweist, d.h.

$$
\begin{aligned}
\boldsymbol{G}_{\boldsymbol{y}_{i} \boldsymbol{\eta}_{i}}(s) & =\boldsymbol{C}_{i}\left(s \boldsymbol{I}_{n_{i}}-\boldsymbol{A}_{i}+\boldsymbol{B}_{i} \boldsymbol{K}_{i}\right)^{-1} \boldsymbol{B}_{i} \boldsymbol{F}_{i} \\
& =\operatorname{diag}\left(g_{i, 1}(s), \ldots, g_{i, p}(s)\right) .
\end{aligned}
$$

Die einzelnen Übertragungsfunktionen $g_{i, k}(s)$, mit $k \in$ $\{1, \ldots, p\}$, werden hierbei als reine Verzögerungsglieder angesetzt:

$$
g_{i, k}(s)=\frac{q_{i, k, 0}}{q_{i, k, 0}+q_{i, k, 1} \cdot s+q_{i, k, 2} \cdot s^{2}+\ldots+s^{\delta_{i, k}}} .
$$

Die Parameter $q_{i, k, l}$ können beliebig vorgegeben werden. Einzig die Differenzordnung $\delta_{i, k}$ ist durch die ungeregelte Strecke bereits eindeutig festgelegt und lässt sich durch eine statische Zustandsregelung nicht verändern. Sie ist definiert als [6]

$$
\delta_{i, k}=\min _{z}\left\{z \geq 1: \boldsymbol{c}_{i, k}^{\top} \boldsymbol{A}_{i}^{z-1} \boldsymbol{B}_{i} \neq \mathbf{0}^{\top}\right\} .
$$


Man bezeichnet $\delta_{i, k}$ auch als Differenzordnung bezüglich der Ausgangsgröße $y_{i, k}$ und $\delta_{i}=\sum_{k=1}^{p} \delta_{i, k}$ als Differenzordnung des Systems. Aus (11) lässt sich die Beziehung

$$
\begin{array}{r}
q_{i, k, 0} \cdot \eta_{i, k}(s)=q_{i, k, 0} \cdot y_{i, k}(s)+q_{i, k, 1} \cdot s \cdot y_{i, k}(s)+\ldots \\
\ldots+s^{\delta_{i, k}} \cdot y_{i, k}(s)
\end{array}
$$

ableiten, die im Zeitbereich in die Differentialgleichung

$$
q_{i, k, 0} \cdot \eta_{i, k}=q_{i, k, 0} \cdot y_{i, k}+q_{i, k, 1} \cdot \dot{y}_{i, k}+\ldots+\stackrel{\left(\delta_{i, k}\right)}{y_{i, k}}
$$

übergeht. Unter Berücksichtigung von $y_{i, k}=\boldsymbol{c}_{i, k}^{\top} \boldsymbol{x}_{i}$ und (12) folgt für die einzelnen Ableitungen von $y_{i, k}$

$$
\begin{aligned}
& \dot{y}_{i, k}=\boldsymbol{c}_{i, k}^{\top} \boldsymbol{A}_{i} \boldsymbol{x}_{i}+\underbrace{\boldsymbol{c}_{i, k}^{\top} \boldsymbol{B}_{i}}_{=\mathbf{0}} \boldsymbol{u}_{i}, \\
& \ddot{y}_{i, k}=\boldsymbol{c}_{i, k}^{\top} \boldsymbol{A}_{i}^{2} \boldsymbol{x}_{i}+\underbrace{\boldsymbol{c}_{i, k}^{\top} A_{i} \boldsymbol{B}_{i}}_{=\mathbf{0}} \boldsymbol{u}_{i}, \\
& y_{i, k}^{\left(\delta_{i, k}\right)}=\boldsymbol{c}_{i, k}^{\top} \boldsymbol{A}_{i}^{\delta_{i, k}} \boldsymbol{x}_{i}+\underbrace{\boldsymbol{c}_{i, k}^{\top} \boldsymbol{A}_{i, k}^{\delta_{i, k}} \boldsymbol{B}_{i}}_{=\boldsymbol{d}_{i, k}^{\star \top}} \boldsymbol{u}_{i} .
\end{aligned}
$$

Durch Einsetzen von (14) in (13) ergibt sich der Zusammenhang

$$
\boldsymbol{c}_{i, k}^{\top}\left(\boldsymbol{A}_{i}^{\delta_{i, k}}+\sum_{l=0}^{\delta_{i, k}-1} q_{i, k, l} \boldsymbol{A}_{i}^{l}\right) \boldsymbol{x}_{i}+\boldsymbol{d}_{i, k}^{\star \top} \boldsymbol{u}_{i}=q_{i, k, 0} \cdot \eta_{i, k} .
$$

Fasst man nun diesen Ausdruck für alle $k \in\{1, \ldots, p\}$ in Matrixschreibweise zusammen, folgt

$$
D_{i}^{\star} u_{i}=-M_{i} x_{i}+N_{i} \eta_{i}
$$

$$
\begin{aligned}
& \operatorname{mit} \boldsymbol{D}_{i}^{\star}= {\left[\boldsymbol{d}_{i, 1}^{\star} \cdots \boldsymbol{d}_{i, p}^{\star}\right]^{\top} \in \mathbb{R}^{p \times m_{i}} \text { und } } \\
& \boldsymbol{M}_{i}=\left[\begin{array}{c}
\boldsymbol{c}_{i, 1}^{\top}\left(\boldsymbol{A}_{i}^{\delta_{i, 1}}+\sum_{l=0}^{\delta_{i, 1}-1} q_{i, 1, l} \boldsymbol{A}_{i}^{l}\right) \\
\vdots \\
\boldsymbol{c}_{i, p}^{\top}\left(\boldsymbol{A}_{i}^{\delta_{i, p}}+\sum_{l=0}^{\delta_{i, p}-1} q_{i, p, l} \boldsymbol{A}_{i}^{l}\right)
\end{array}\right] \in \mathbb{R}^{p \times n_{i}}, \\
& \boldsymbol{N}_{i}=\operatorname{diag}\left(q_{i, 1,0}, \ldots, q_{i, p, 0}\right) \in \mathbb{R}^{p \times p} .
\end{aligned}
$$

Aus (15) ist ersichtlich, dass für rang $\left(D_{i}^{\star}\right)=p$ ein Regelgesetz

$$
\boldsymbol{u}_{i}=-\boldsymbol{K}_{i} \boldsymbol{x}_{i}+\boldsymbol{F}_{i} \boldsymbol{\eta}_{i}
$$

mit $\boldsymbol{K}_{i}=\boldsymbol{D}_{i}^{\star+} \boldsymbol{M}_{i}$ und $\boldsymbol{F}_{i}=\boldsymbol{D}_{i}^{\star+} \boldsymbol{N}_{i}$, existiert, sodass sich eine Entkopplung gemäß (10) mit den Übertragungsfunktionen (11) einstellt. Dabei können über die Parameter $q_{i, k, l}$, die in den Matrizen $\boldsymbol{M}_{i}$ und $\boldsymbol{N}_{i}$ stecken, die Polstellen der Übertragungsfunktionen $g_{i, k}(s)$ beliebig vorgegeben werden.
Anzumerken sei, dass im Fall von $\delta_{i}<n_{i}$ der geschlossene Regelkreis weniger Eigenwerte aufweist als die ungeregelte Strecke. Dies ist jedoch nur dann möglich, wenn durch den Entkopplungsregler $n_{i}-\delta_{i}$ Eigenwerte unter die invarianten Nullstellen des Systems geschoben und dadurch unbeobachtbar gemacht werden. Damit also eine stabile Entkopplung mit dem statischen Zustandsregler (9) möglich ist, muss sinnvollerweise vorausgesetzt werden, dass das System (1) keine invarianten Nullstellen in der geschlossenen rechten $s$-Halbebene aufweist. Des Weiteren ist zu beachten, dass für quadratische Systeme, d.h. für Systeme mit gleicher Anzahl von Ein- und Ausgängen, die sogenannte Entkoppelbarkeitsbedingung rang $\left(\boldsymbol{D}_{i}^{\star}\right)=$ $p$ notwendig und hinreichend für die Entkoppelbarkeit des Systems (1) ist [6]. Für überaktuierte Systeme, d.h. für Systeme mit mehr Ein- als Ausgängen, ist diese Bedingung allerdings nur hinreichend [2]. Im Fall von $\delta_{i}<n_{i}$ kann es für überaktuierte Systeme vorkommen, dass eine ungeeignete Wahl der Rechtsinversen $\boldsymbol{D}_{i}^{\star+}$ zu instabilen Eigenwerten führt, auch wenn keine invarianten Nullstellen in der rechten $s$-Halbebene liegen. Allerdings existiert in diesem Fall immer eine Rechtsinverse von $\boldsymbol{D}_{i}^{\star}$, die eine stabile Entkopplung gewährleistet [26]. Zusammenfassend werden folgende Annahmen bezüglich der Agenten (1) getroffen.

Annahme 1. Die Matrizen $\left(\boldsymbol{A}_{i}, \boldsymbol{B}_{i}\right)$ sind vollständig steuerbar.

Annahme 2. Die invarianten Nullstellen von $\left(\boldsymbol{A}_{i}, \boldsymbol{B}_{i}, \boldsymbol{C}_{i}\right)$ liegen alle in der offenen linken $s$-Halbebene und die Matrix $\boldsymbol{D}_{i}^{\star}$ ist invertierbar.

Während Annahme 1 sicherstellt, dass alle Eigenwerte beliebig platziert werden können, garantiert Annahme 2 eine stabile Entkopplung durch die statische Zustandsregelung (9).

Anmerkung 1. Weist ein System invariante Nullstellen in der rechten $s$-Halbebene auf oder ist die Entkoppelbarkeitsbedingung verletzt, so ist im Allgemeinen eine Entkopplung mit einem statischen Zustandsregler nicht möglich. In diesem Fall muss auf eine dynamische Rückführung zurückgegriffen werden, wie z.B. in [16] ausgeführt.

\subsection{Synchronisierungsregler}

In diesem Abschnitt wird eine Methode präsentiert, um mit Hilfe des Entkopplungsentwurfs die Agenten (1) auf eine gemeinsame Trajektorie zu synchronisieren. $\mathrm{Zu}$ diesem Zweck wird zunächst angenommen, dass für jeden Übertragungspfad die Differenzordnung über alle Agenten 
gleich ist, d.h. $\delta_{i, k}=\delta_{k}, \forall i \in\{1, \ldots, N\}$. Außerdem wird vorausgesetzt, dass diese Differenzordnung nicht kleiner ist als die Ordnung $\bar{n}_{k}$ eines Wunschpolynoms $\bar{a}_{k}(s)$, also $\delta_{k} \geq \bar{n}_{k}$. Dieses Wunschpolynom beschreibt dabei die Eigenbewegungen der angestrebten Synchronisierungstrajektorie.

Hat man die Matrizen $\boldsymbol{K}_{i}$ und $\boldsymbol{F}_{i}$ des Regelgesetzes (9) gemäß Abschnitt 3.1 ausgelegt, ergibt sich in jedem Übertragungspfad des geschlossenen Regelkreises ein SISOSystem mit der Übertragungsfunktion (11). Hierbei lassen sich die Parameter $q_{i, k, l}$ frei vorgeben. Diese Parameter werden nun für alle Agenten identisch und darüber hinaus so gewählt, dass der Nenner von (11) das Wunschpolynom $\bar{a}_{k}(s)$ enthält. Dies führt zu

$$
g_{i, k}(s)=g_{k}(s)=\frac{q_{k, 0}}{\bar{a}_{k}(s) \cdot \widetilde{a}_{k}(s)}, \forall i \in\{1, \ldots, N\},
$$

wobei $\widetilde{a}_{k}(s)$ ein beliebiges Hurwitz-Polynom darstellt. Eine Festlegung gemäß (16) ist möglich, da vorausgesetzt wurde, dass die Differenzordnung für alle Agenten gleich und nicht kleiner als die Ordnung des Wunschpolynoms ist. Führt man obige Vorgehensweise für alle Übertragungspfade durch, so ist das gesamte Ein-/Ausgangsverhalten für alle Agenten identisch.

Anmerkung 2. Ist das Polynom $\widetilde{a}_{k}(s)$ Hurwitz, werden die zugehörigen Eigenbewegungen asymptotisch abklingen, sodass die Dynamik von $y_{i, k}$ durch die Eigenbewegungen des Wunschpolynoms $\bar{a}_{k}(s)$ bestimmt wird.

Nachdem das Ein-/Ausgangsverhalten der Agenten homogenisiert ist, stellt sich die Frage, wie eine Synchronisierung der Ausgänge erreicht werden kann. Auch wenn das Übertragungsverhalten der Agenten gleich ist, sind ihre internen Zustände und ihre Systemmatrizen nicht homogen. Um dieses Problem zu lösen, wird für jede Übertragungsfunktion (16) eine Zustandsraumdarstellung

$$
\begin{aligned}
\dot{\boldsymbol{x}}_{i, k} & =\widehat{\boldsymbol{A}}_{k} \widehat{\boldsymbol{x}}_{i, k}+\widehat{\boldsymbol{b}}_{k} \eta_{i, k}, \\
y_{i, k} & =\widehat{\boldsymbol{c}}_{k}^{\top} \widehat{\boldsymbol{x}}_{i, k},
\end{aligned}
$$

mit den virtuellen Zuständen $\widehat{\boldsymbol{x}}_{i, k} \in \mathbb{R}^{\delta_{k}}$, eingeführt. Das System $\left(\widehat{A}_{k}, \widehat{\boldsymbol{b}}_{k}, \widehat{\boldsymbol{c}}_{k}^{\top}\right)$ wird dabei in Regelungsnormalform angesetzt. Da die Systembeschreibung in (17) für alle Agenten identisch ist, lassen sich die Ergebnisse aus Abschnitt 2.4 darauf anwenden. Mit Hilfe von Lemma 1 kann ein Reglervektor $\boldsymbol{h}_{k}^{\top} \in \mathbb{R}^{1 \times \delta_{k}}$ ermittelt werden, der zusammen mit dem Regelgesetz

$$
\eta_{i, k}=-\boldsymbol{h}_{k}^{\top} \sum_{j=1}^{N} a_{\mathcal{G}_{j i}}\left(\widehat{\boldsymbol{x}}_{i, k}-\widehat{\boldsymbol{x}}_{j, k}\right)
$$

dafür sorgt, dass sich die Zustände $\widehat{\boldsymbol{x}}_{i, k}$ und dadurch auch die Ausgänge $y_{i, k}$ synchronisieren.
Das Problem besteht allerdings darin, dass für das Regelgesetz (18) die unbekannten virtuellen Zustände $\widehat{\boldsymbol{x}}_{i, k}$ benötigt werden. Diese Zustände lassen sich jedoch über eine geeignete Zustandstransformation ermitteln. Aufgrund der Regelungsnormalform von (17) gilt für den Zustandsvektor $\widehat{\boldsymbol{x}}_{i, k}$ die Beziehung

$$
\widehat{\boldsymbol{x}}_{i, k}=\left[\begin{array}{c}
\widehat{x}_{i, k, 1} \\
\widehat{x}_{i, k, 2} \\
\vdots \\
\widehat{x}_{i, k, \delta_{k}}
\end{array}\right]=\frac{1}{q_{k, 0}} \cdot\left[\begin{array}{c}
y_{i, k} \\
\dot{y}_{i, k} \\
\vdots \\
\left(\delta_{k}-1\right) \\
y_{i, k}
\end{array}\right] .
$$

Der virtuelle Vektor $\widehat{\boldsymbol{x}}_{i, k}$ setzt sich also aus der Ausgangsgröße $y_{i, k}$ sowie ihre zeitlichen Ableitungen bis zur $\left(\delta_{k}-\right.$ 1)-ten Ableitung zusammen. Aus (14) ist bereits bekannt, dass für die Ausgangsgröße und ihre zeitlichen Ableitungen folgender Zusammenhang gilt

$$
\frac{1}{q_{k, 0}} \cdot\left[\begin{array}{c}
y_{i, k} \\
\dot{y}_{i, k} \\
\vdots \\
\left(\delta_{k}-1\right) \\
y_{i, k}
\end{array}\right]=\frac{1}{q_{k, 0}} \cdot\left[\begin{array}{c}
\boldsymbol{c}_{i, k}^{\top} \\
\boldsymbol{c}_{i, k}^{\top} \boldsymbol{A}_{i} \\
\vdots \\
\boldsymbol{c}_{i, k}^{\top} \boldsymbol{A}_{i}^{\delta_{k}-1}
\end{array}\right] \boldsymbol{x}_{i}=\boldsymbol{T}_{i, k} \boldsymbol{x}_{i},
$$

mit $T_{i, k} \in \mathbb{R}^{\delta_{k} \times n_{i}}$. Demnach ergeben sich die virtuellen Zustände für das System (17) aus der Transformation $\widehat{\boldsymbol{x}}_{i, k}=\boldsymbol{T}_{i, k} \boldsymbol{x}_{i}$. Fasst man nun die virtuellen Zustände jedes Übertragungspfads in einem gemeinsamen Vektor $\widehat{\boldsymbol{x}}_{i}^{\top}=\left[\widehat{\boldsymbol{x}}_{i, 1}^{\top} \cdots \hat{\boldsymbol{x}}_{i, p}^{\top}\right] \in \mathbb{R}^{\delta}$ zusammen, so ergibt sich mit $\boldsymbol{H}=$ $\operatorname{diag}\left(\boldsymbol{h}_{1}^{\top}, \ldots, \boldsymbol{h}_{p}^{\top}\right) \in \mathbb{R}^{p \times \delta}$ das Regelgesetz

$$
\boldsymbol{\eta}_{i}=-\boldsymbol{H} \sum_{j=1}^{N} a_{\mathcal{S}_{j i}}\left(\widehat{\boldsymbol{x}}_{i}-\widehat{\boldsymbol{x}}_{j}\right),
$$

das dafür sorgt, dass sich die virtuellen Zustände $\widehat{\boldsymbol{x}}_{i}$ und damit die Ausgangsgrößen $\boldsymbol{y}_{i}$ synchronisieren.

Anstelle des Regelgesetzes (19) kann auch alternativ das Regelgesetz

$$
\eta_{i}=-\sum_{j=1}^{N} a_{\mathcal{G}_{j i}}\left(\boldsymbol{\mu}_{i}-\boldsymbol{\mu}_{j}\right),
$$

mit $\boldsymbol{\mu}_{i}=\boldsymbol{H} \hat{\boldsymbol{x}}_{i} \in \mathbb{R}^{p}$, verwendet werden. Offensichtlich sind beide Regelgesetze identisch. Allerdings weist (20) den Vorteil auf, dass der Kommunikationsaufwand reduziert wird. Während in (19) der Austausch eines Signals der Länge $\delta$ erfordert wird, muss in (20) lediglich ein Signal der Länge $p$ kommuniziert werden. Da im Allgemeinen $\delta \geq p$ gilt, wird der Kommunikationsaufwand dadurch verringert. 


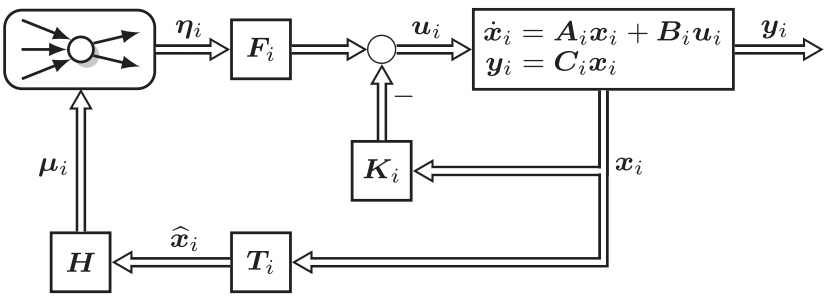

Abbildung 2: Reglerstruktur bestehend aus Entkopplungs- und Synchronisierungsregler. Der Vektor $\boldsymbol{\mu}_{i}$ wird über das Netzwerk kommuniziert.

Zusammenfassend lässt sich der Entwurf aus Entkopplungsregler und Synchronisierungsregler wie in Abbildung 2 dargestellt zusammenfassen. Hierbei gilt für die Transformationsmatrix $\boldsymbol{T}_{i}=\left[\boldsymbol{T}_{i, 1}^{\top} \cdots \boldsymbol{T}_{i, p}^{\top}\right]^{\top}$.

Durch die präsentierte Vorgehensweise lassen sich die Ausgänge von Agenten beliebiger Heterogenität durch die statischen Regelgesetze (9) und (20) synchronisieren, gesetzt den Fall, dass die Annahmen 1 und 2 erfüllt sind. Dabei wurde sinnvollerweise vorausgesetzt, dass die Differenzordnungen bezüglich der Ausgänge $y_{i, k}$ nicht kleiner als die Ordnungen der Wunschpolynome $\bar{a}_{k}(s)$ sind. Andernfalls könnte durch den statischen Entkopplungsregler diese Dynamik nicht vorgegeben werden. Außerdem wurde angenommen, dass für jeden Übertragungspfad die Differenzordnungen der Agenten gleich sind, da sonst die Polynome $\widetilde{a}_{k}(s)$ unterschiedliche Dimensionen aufweisen und die Systeme (17) somit nicht homogen sind.

Anmerkung 3. Offensichtlich beschreibt $\widetilde{a}_{k}(s)$ eine Dynamik, die sich nur im Fall von $\delta_{k}>\bar{n}_{k}$ ergibt und für die Synchronisierungstrajektorie nicht benötigt wird. Durch die Festlegung des Wunschpolynoms $\bar{a}_{k}(s)$ ist das InnereModell-Prinzip bereits erfüllt und eine Synchronisierung theoretisch möglich. Deshalb ist es prinzipiell nicht notwendig das Polynom $\widetilde{a}_{k}(s)$ zu homogenisieren. Allerdings wäre dann die Systembeschreibung (17) nicht mehr identisch für alle Agenten und Satz 1 somit nicht anwendbar. Insbesondere für Kommunikationstopologien mit Kreisstrukturen würden sich die Analyse und der Reglerentwurf dadurch erheblich verkomplizieren. Diese Tatsache wird z.B. auch in [17] erkannt.

Die Forderung nach gleicher Differenzordnung ist aus praktischer Sicht nicht unrealistisch, insbesondere da die Agenten in einem heterogenen Multi-Agenten-System häufig eine ähnliche strukturelle Dynamik aufweisen. Beispielsweise erfolgt die Kraftübertragung für verschiedene Fahrzeuge i.d.R. auf gleiche Art, sodass die Agenten innerhalb einer heterogenen Fahrzeugkolonne die gleiche Differenzordnung aufweisen. In diesem Fall kann mit Hilfe der beschriebenen, rein statischen, Regelungsstra- tegie eine Ausgangssynchronisierung mit einer vorgebbaren Wunschdynamik erzielt werden, vorausgesetzt $\delta_{k} \geq$ $\bar{n}_{k}$. Sind die Anforderungen an die Differenzordnungen jedoch nicht erfüllt, muss von einer statischen Regelungsstrategie auf eine dynamische übergegangen werden, indem eine Anpassung der Differenzordnungen vorgenommen wird.

\subsection{Anpassung der Differenzordnungen}

Für den Fall, dass in einem Übertragungspfad $\delta_{i, k} \neq \delta_{j, k}$ oder $\delta_{i, k}<\bar{n}_{k}$ gilt, ist es notwendig die Differenzordnung in diesem Übertragungspfad zu korrigieren. Dies kann durch das Vorschalten eines Verzögerungsgliedes erreicht werden, sodass $\delta_{i, k}=\bar{\delta}_{k} \geq \bar{n}_{k}$ für alle Agenten $i \in\{1, \ldots, N\}$ und alle Übertragungspfade $k \in\{1, \ldots, p\}$ gilt. Dabei ist

$$
\bar{\delta}_{k}= \begin{cases}\max _{i}\left\{\delta_{i, k}\right\}, & \text { falls } \bar{n}_{k}<\max _{i}\left\{\delta_{i, k}\right\}, \\ \bar{n}_{k}, & \text { falls } \bar{n}_{k} \geq \max _{i}\left\{\delta_{i, k}\right\} .\end{cases}
$$

Wenn für den $i$-ten Agenten im $k$-ten Übertragungspfad $\delta_{i, k}<\bar{\delta}_{k}$ gilt, muss ein Verzögerungsglied der Ordnung $z_{i, k}=\bar{\delta}_{k}-\delta_{i, k}$ mit der Zustandsraumdarstellung

$$
\begin{aligned}
\dot{\boldsymbol{x}}_{i, k}^{v} & =\boldsymbol{A}_{i, k}^{v} \boldsymbol{x}_{i, k}^{v}+\boldsymbol{b}_{i, k}^{v} \zeta_{i, k}, \\
\eta_{i, k} & =\boldsymbol{c}_{i, k}^{v \top} \boldsymbol{x}_{i, k}^{v},
\end{aligned}
$$

$\boldsymbol{x}_{i, k}^{v} \in \mathbb{R}^{z_{i, k}}$, vorgeschaltet werden. Hierbei bezeichnet $\zeta_{i, k}$ das Eingangssignal des Verzögerungsgliedes und wird weiter unten definiert. Die Zustandsraumdarstellung dieses Verzögerungsgliedes wird so parametriert, dass für seine Übertragungsfunktion $g_{i, k}^{v}(s)$ die Beziehung

$$
g_{i, k}^{v}(s) \cdot g_{i, k}(s)=g_{k}(s)
$$

gilt, wobei $g_{k}(s)$ wie in (16) gegeben ist. Wird nun für die resultierende Übertragungsfunktion (23) eine virtuelle Zustandsraumdarstellung gewählt, deren interne Zustände wie in Abschnitt 3.2 ausgeführt - synchronisiert werden, so werden sich auch die zugehörigen Ausgangsgrößen $y_{i, k}$ synchronisieren.

Problematisch bei dieser Vorgehensweise ist jedoch, dass je nach Wahl des Wunschpolynoms $\bar{a}_{k}(s)$ die Übertragungsfunktionen $g_{i, k}^{v}(s)$ und $g_{i, k}(s)$ einen von Null verschiedenen Imaginäranteil aufweisen können und dadurch praktisch nicht realisierbar sind. Dies soll an folgendem Beispiel verdeutlicht werden. Angenommen die Differenzordnung von Agent $i$ bezüglich des Ausgangs $y_{i, k}$ ist $\delta_{i, k}=1$ und das Wunschpolynom ist $\mathrm{zu} \bar{a}_{k}(s)=s^{2}+$ 1 festgelegt. Dann wird ein Verzögerungsglied der Ordnung $z_{i, k}=1$ benötigt. Nach der obigen Herangehensweise müsste beim Entkopplungsentwurf der Nenner der 
Übertragungsfunktion $g_{i, k}(s)$ entweder zu $s+j$ oder $s-j$ gewählt werden und der Nenner der Übertragungsfunktion $g_{i, k}^{v}(s)$ entsprechend zu $s-j$ oder $s+j$. Nur dann ist gewährleistet, dass $(s+j) \cdot(s-j)=s^{2}+1$. Diese imaginären Polstellen lassen sich jedoch durch eine reelle Regelung nicht einzeln realisieren. Um dieses Problem zu umgehen, wird anstelle des Ansatzes (23) ein alternativer Lösungsweg vorgestellt.

Weist ein Agent in einem Übertragungspfad eine Differenzordnung von $\delta_{i, k}<\bar{\delta}_{k}$ auf, werden beim Entkopplungsentwurf die Parameter $q_{i, k, l}$ und das Verzögerungsglied so ausgelegt, dass die Gesamtübertragungsfunktion (23) eine Integriererkette darstellt. Die zugehörige $\mathrm{Zu}$ standsraumbeschreibung kann zu

$$
\begin{aligned}
\dot{\boldsymbol{x}}_{i, k}^{\star} & =\left[\begin{array}{cccc}
0 & 1 & \cdots & 0 \\
\vdots & \vdots & \ddots & \vdots \\
0 & 0 & \cdots & 1 \\
0 & 0 & \cdots & 0
\end{array}\right] \boldsymbol{x}_{i, k}^{\star}+\left[\begin{array}{c}
0 \\
\vdots \\
0 \\
1
\end{array}\right] \zeta_{i, k}, \\
y_{i, k} & =\left[\begin{array}{llll}
q_{k, 0} & 0 & \cdots & 0
\end{array}\right] \boldsymbol{x}_{i, k}^{\star},
\end{aligned}
$$

mit $\boldsymbol{x}_{i, k}^{\star \top}=\left[\widehat{\boldsymbol{x}}_{i, k}^{\top} \boldsymbol{x}_{i, k}^{\nu \top}\right] \in \mathbb{R}^{\bar{\delta}_{k}}$, angesetzt werden. Für dieses Gesamtsystem wird nun eine Regelung

$$
\zeta_{i, k}=-\boldsymbol{k}_{i, k}^{\star \top} \boldsymbol{x}_{i, k}^{\star}+\eta_{i, k}^{\star}
$$

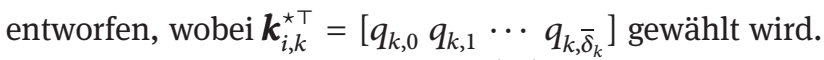
Dadurch wird dem Gesamtsystem (24) das Wunschverhalten gemäß (16) aufgeprägt. Da der geschlossene Regelkreis aus (24) und (25) das Wunschverhalten aufweist und in Regelungsnormalform vorliegt, entspricht es der Systembeschreibung (17). Für dieses System wurde allerdings bereits gezeigt, dass mit Hilfe des Regelgesetzes (18) Synchronisierung erreicht wird. Dementsprechend muss in (25) $\eta_{i, k}^{\star} \mathrm{zu}$

$$
\eta_{i, k}^{\star}=-\sum_{j=1}^{N} a_{\mathcal{G}_{j i}}\left(\mu_{i, k}^{\star}-\mu_{j, k}^{\star}\right)
$$

gewählt werden, wobei $\mu_{i, k}^{\star}=\boldsymbol{h}_{k}^{\top} \boldsymbol{x}_{i, k}^{\star}$.

Abbildung 3 fasst die Ausführungen dieses Abschnitts zusammen. Hierbei wird der Ausschnitt für die Anpassung der Differenzordnung für den $k$-ten Übertragungspfad angezeigt. Die Variable $\eta_{i, k}$ beschreibt das $k$-te Element des Vektors $\boldsymbol{\eta}_{i}$, welcher die Eingangsgröße für den Entkopplungsregler (9) darstellt. Falls für ein Element dieses Vektors keine Anpassung der Differenzordnung vorgenommen werden muss, so erfolgt der Entwurf für diesen Übertragungspfad gemäß den Ausführungen von Abschnitt 3.2.

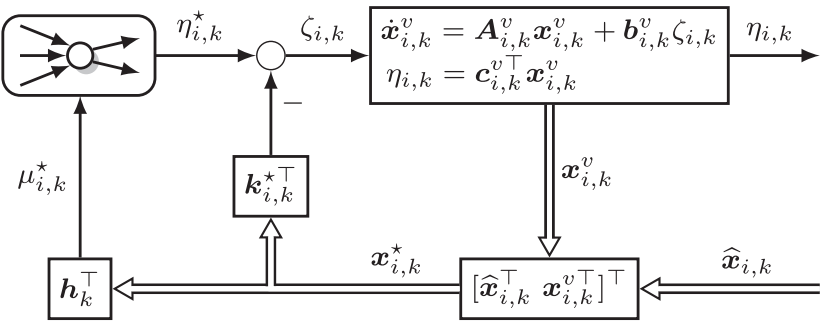

Abbildung 3: Reglerstruktur zur Anpassung der Differenzordnung von Agent $i$ bezüglich des Ausgangs $y_{i, k}$.

\section{Exakte Trajektorienfolge}

Die beschriebene Regelungsstrategie ermöglicht es nicht nur die Dynamik der Synchronisierungstrajektorie vorzugeben, sondern auch mit Hilfe einer simplen Erweiterung einer vorgegebenen Wunschtrajektorie exakt zu folgen. Hierzu kann für jede Ausgangsgröße $k \in\{1, \ldots, p\}$ ein autonomes System der Form

$$
\begin{aligned}
& \dot{\overline{\boldsymbol{x}}}_{k}=\overline{\boldsymbol{A}}_{k} \overline{\boldsymbol{x}}_{k}, \\
& \bar{y}_{k}=\overline{\boldsymbol{c}}_{k}^{\top} \overline{\boldsymbol{x}}_{k},
\end{aligned}
$$

mit $\overline{\boldsymbol{x}}_{k} \in \mathbb{R}^{\bar{n}_{k}}$, aufgestellt werden. Die Ausgangsgröße $\bar{y}_{k}$ beschreibt die Wunschtrajektorie, der die Agenten folgen sollen. Die Matrix $\overline{\boldsymbol{A}}_{k}$ wird dabei so angesetzt, dass ihr charakteristisches Polynom dem Wunschpolynom $\bar{a}_{k}(s)$ in (16) entspricht, bzw. das Wunschpolynom $\bar{a}_{k}(s)$ wird so angesetzt, dass es dem charakteristischen Polynom von $\bar{A}_{k}$ entspricht. Die Notwendigkeit hierfür zeigt sich später. Um triviale Lösungen auszuschließen, wird angenommen, dass $\overline{\boldsymbol{A}}_{k}$ keine Hurwitz-Matrix ist.

Wird nun für einen Agenten $\tau$, der einen Wurzelknoten im Kommunikationsnetzwerk darstellt, eine Folgeregelung entworfen, sodass

$$
\lim _{t \rightarrow \infty}\left|y_{\tau, k}(t)-\bar{y}_{k}(t)\right|=0,
$$

dann synchronisieren alle Agenten zur Wunschtrajektorie $\bar{y}_{k}$, wie nachfolgend erläutert wird.

Aus Abschnitt 3 ist bekannt, dass sich die Dynamik der Ausgangsgröße $y_{\tau, k}$ gemäß (17) mit Hilfe der homogenisierten Systemdarstellung $\left(\widehat{\boldsymbol{A}}_{k}, \widehat{\boldsymbol{b}}_{k}, \widehat{\boldsymbol{c}}_{k}^{\top}\right)$ beschreiben lässt. Stellt Agent $\tau$ ein Wurzelknoten im Netzwerk dar, kann für diesen Agenten das Regelgesetz (18) zu $\eta_{\tau, k}=0$ gesetzt werden. Dies ist äquivalent dazu, dass für Agent $\tau$ alle eingehenden Kanten im zugehörigen Kommunikationsgraph entfernt werden. Da Agent $\tau$ ein Wurzelknoten darstellt, enthält der Graph weiterhin einen gerichteten Spannbaum, bei dem Agent $\tau$ explizit als Anführer gewählt wurde. Wenn nun alle Agenten synchronisieren und 
darüber hinaus eine Trajektorienfolge gemäß (28) für den Wurzelagenten $\tau$ erreicht ist, bedeutet das, dass alle Agenten der Wunschtrajektorie exakt folgen. Es ist zwar nicht notwendig $\eta_{\tau, k}=0 \mathrm{zu}$ setzen, dies ist allerdings sinnvoll, da Agent $\tau$ als Anführer die Synchronisierungstrajektorie für alle anderen Agenten vorgeben soll. Stattdessen wird für Agent $\tau$ ein Regelgesetz $\bar{\eta}_{\tau, k}$ gesucht, sodass (28) erreicht wird. Das nachfolgende Lemma, welches hier ohne Beweis angegeben wird, fasst den Entwurf zusammen.

Lemma 2 ([23], Theorem 2.3.1). Das Regelgesetz

$$
\bar{\eta}_{\tau, k}=\boldsymbol{\Gamma}_{k} \overline{\boldsymbol{x}}_{k}-\overline{\boldsymbol{h}}_{k}^{\top}\left(\widehat{\boldsymbol{x}}_{\tau, k}-\boldsymbol{\Pi}_{k} \overline{\boldsymbol{x}}_{k}\right)
$$

führt zu einer Trajektorienfolge gemäß (28) genau dann, wenn die Matrixgleichungen

$$
\begin{aligned}
\boldsymbol{\Pi}_{k} \overline{\boldsymbol{A}}_{k} & =\widehat{\boldsymbol{A}}_{k} \boldsymbol{\Pi}_{k}+\widehat{\boldsymbol{b}}_{k} \boldsymbol{\Gamma}_{k}, \\
\overline{\boldsymbol{c}}_{k}^{\top} & =\widehat{\boldsymbol{c}}_{k}^{\top} \boldsymbol{\Pi}_{k},
\end{aligned}
$$

für ein $\Pi_{k} \in \mathbb{R}^{\delta_{k} \times \bar{n}_{k}}$ und $\Gamma_{k} \in \mathbb{R}^{1 \times \bar{n}_{k}}$, lösbar sind. Der Reglervektor $\overline{\boldsymbol{h}}_{k}^{\top}$ muss dabei so ausgelegt werden, dass $\left(\widehat{\boldsymbol{A}}_{k}-\widehat{\boldsymbol{b}}_{k} \overline{\boldsymbol{h}}_{k}^{\top}\right)$ Hurwitz ist.

Anmerkung 4. Falls für Agent $\tau$ bezüglich der Ausgangsgröße $y_{\tau, k}$ eine Anpassung der Differenzordnung notwendig ist, muss anstelle von (18) das Regelgesetz (26) zu $\eta_{i, k}^{\star}=$ 0 gesetzt und der Zustandsvektor $\widehat{\boldsymbol{x}}_{\tau, k}$ in (29) durch $\boldsymbol{x}_{\tau, k}^{\star}$ ersetzt werden.

Lemma 2 stellt ein bekanntes Resultat aus der sogenannten output regulation theory [8] dar, weshalb an dieser Stelle auf eine Beweisführung verzichtet wird.

Das Regelgesetz (29) lässt sich wie folgt interpretieren. Der erste Teil des Regelgesetzes dient als Vorsteuerung, die es dem geschlossenen Regelkreis ermöglicht den Verlauf der Wunschtrajektorie nachzubilden. Der zweite Teil des Regelgesetzes sorgt dafür, dass Abweichungen von dieser Wunschtrajektorie asymptotisch abklingen. Beim Entkopplungsentwurf wurde die Matrix $\widehat{\boldsymbol{A}}_{k}$ so angesetzt, dass es das charakteristische Polynom $\bar{a}_{k}(s)$ und somit die Eigenbewegungen von $\bar{A}_{k}$ enthält. Aus diesem Grund ist eine Vorsteuerung nicht notwendig, um den Verlauf der Wunschtrajektorie zu erzeugen, sodass das Ergebnis von (30) stets $\boldsymbol{\Gamma}_{k}=\mathbf{0}$ liefert. In [17] wird diese Tatsache unter dem Begriff system intersection zusammengefasst. Es wird gezeigt, dass wenn die Eigenbewegungen eines Systems in einem anderen System enthalten sind, dann existieren immer Anfangswerte $\widehat{\boldsymbol{x}}_{\tau, k}(0)$ und $\overline{\boldsymbol{x}}_{k}(0)$, sodass die Ausgänge beider Systeme für $t \geq 0$ identisch sind. Dementsprechend kann anstelle des Regelgesetzes (29)

$$
\bar{\eta}_{\tau, k}=-\overline{\boldsymbol{h}}_{k}^{\top}\left(\widehat{\boldsymbol{x}}_{\tau, k}-\boldsymbol{\Pi}_{k} \overline{\boldsymbol{x}}_{k}\right)
$$

angesetzt werden. Gemäß den obigen Überlegungen ist dieses Regelgesetz nur dann aktiv, wenn zwischen $y_{i, k}$ und $\bar{y}_{k}$ eine Abweichung besteht. Nachdem diese Abweichung ausgeregelt wurde und sich (28) eingestellt hat, folgt $\bar{\eta}_{\tau, k}=$ 0 . Unter Berücksichtigung dieser Tatsache kann folgende Schlussfolgerung gezogen werden. Hat sich der Wurzelagent $\tau$ zur Wunschtrajektorie synchronisiert, so ist $\bar{\eta}_{\tau, k}=$ 0 und Agent $\tau$ agiert als ein autonomes System, das exakt die Ausgangstrajektorie von (27) produziert. Zusätzlich synchronisieren alle anderen Agenten zu diesem Wurzelagenten, sodass

$$
\lim _{t \rightarrow \infty}\left|y_{i, k}(t)-\bar{y}_{k}(t)\right|=0, \quad \forall i \in\{1, \ldots, N\} .
$$

Anmerkung 5. Wenn die Agenten die Eigenbewegungen von (27) nicht enthalten, wird eine Vorsteuerung gemäß (29) benötigt, um Agent $\tau$ zur Wunschtrajektorie zu synchronisieren. Allerdings können dann alle anderen Agenten nicht zu Agent $\tau$ synchronisieren, solange ihnen die Vorsteuerung $\Gamma_{k} \overline{\boldsymbol{x}}_{k}$ fehlt. Nur mit Hilfe dieser Vorsteuerung wäre eine Synchronisierung noch möglich. Dies würde allerdings in einem zentralisierten Entwurf resultieren, da jeder Agent die Zustände des autonomen Systems (27) kennen müsste. Aus diesem Grund werden beim Entkopplungsentwurf den homogenisierten Agenten die Eigenbewegungen von (27) aufgeprägt, wodurch die Notwendigkeit der Vorsteuerung eliminiert wird.

Anmerkung 6. Die Einführung eines autonomen Systems für die Vorgabe einer gezielten Synchronisierungstrajektorie ist eine bekannte Vorgehensweise für die Synchronisierung heterogener Agenten. Eines der ersten Beiträge hierzu stammt von [27]. Allerdings wird in [27] dieses autonome System für jeden Agenten einzeln eingeführt und jeweils eine Trajektorienfolgeregelung entworfen. Dies resultiert in einer insgesamt hohen Reglerordnung. Im Gegensatz dazu muss in der hier vorgeschlagenen Methode das autonome System nur einmal eingeführt werden, wodurch die Reglerordnung erheblich reduziert werden kann.

Zusammenfassend lässt sich festhalten, dass für einen beliebigen Wurzelagenten im Netzwerk das Regelgesetz (18) bzw. (26) durch das Regelgesetz (31) ersetzt werden muss, um eine exakte Synchronisierung zur Wunschtrajektorie $\bar{y}_{k}$ zu realisieren.

Durch die in diesem Abschnitt beschriebene Vorgehensweise lassen sich im Wesentlichen zwei Erweiterungen erreichen. Gemäß den Ausführungen von Abschnitt 2.4 ergibt sich die Synchronisierungstrajektorie aus einem gewichteten Mittel der Anfangswerte der homogenisierten Agenten. Durch die Einführung des autono- 
men Systems (27) kann jedoch der exakte Verlauf der Synchronisierungstrajektorie über die Anfangswerte $\overline{\boldsymbol{x}}_{k}(0)$ gezielt vorgegeben werden. Darüber hinaus beschränkt sich die Synchronisierungstrajektorie bisher auf Übertragungsfunktionen der Form (16), die als reine Verzögerungsglieder angesetzt werden. Mit Hilfe des autonomen Systems $\left(\overline{\boldsymbol{A}}_{k}, \overline{\boldsymbol{c}}_{k}^{\top}\right)$ können jedoch auch Synchronisierungstrajektorien realisiert werden, die sich in der Form (27) darstellen lassen.

\section{Beispiel}

Zur Veranschaulichung der vorangegangenen Ergebnisse soll als Beispiel die Ausgangssynchronisierung für eine Flotte von $N=4$ verschiedenen Flugzeugmodellen untersucht werden. Hierzu werden die für den Reiseflug linearisierten Lateraldynamiken einer X-29A (Agent 1), Lockheed L-1011 (Agent 2), Boeing-767 (Agent 3) und F-18 HARV (Agent 4) betrachtet. Die Systemmatrizen der Agenten sind aus $[1,3,7]$ und $[24]$ entnommen und lauten

$$
\begin{aligned}
& \boldsymbol{A}_{1}=\left[\begin{array}{cccc}
-0.1645 & 0.0441 & -0.0603 & -0.9928 \\
0 & 0 & 1 & 0.0717 \\
-16.5500 & 0 & -2.5900 & 0.9970 \\
6.7790 & 0 & -0.1023 & -0.0673
\end{array}\right], \\
& \boldsymbol{A}_{2}=\left[\begin{array}{cccc}
-0.1170 & 0.0386 & -0.0003 & -0.9960 \\
0 & 0 & 1 & 0 \\
-5.2000 & 0 & -1 & 0.2490 \\
1.5400 & 0 & -0.0042 & -0.1540
\end{array}\right], \\
& \boldsymbol{A}_{3}=\left[\begin{array}{cccc}
-0.1245 & 0.0414 & 0.0350 & -0.9962 \\
0 & 0 & 1 & 0.0357 \\
-15.2138 & 0.0032 & -2.0587 & 0.6458 \\
1.6447 & -0.0022 & -0.0447 & -0.1416
\end{array}\right], \\
& \boldsymbol{A}_{4}=\left[\begin{array}{cccc}
-0.1703 & 0.0440 & 0.0490 & 0.9980 \\
0 & 0 & 1 & 0.0491 \\
-15.5763 & 0 & -2.3142 & 0.5305 \\
3.0081 & 0 & -0.0160 & -0.1287
\end{array}\right] .
\end{aligned}
$$

Die zugehörigen Eingangsmatrizen sind

$$
\begin{aligned}
& \boldsymbol{B}_{1}=\left[\begin{array}{cc}
0 & 0 \\
0 & 0 \\
1.3470 & 0.2365 \\
0.0919 & -0.0706
\end{array}\right], \\
& \boldsymbol{B}_{2}=\left[\begin{array}{cc}
0 & 0.0200 \\
0 & 0 \\
-1.1200 & 0.3370 \\
-0.0320 & -0.7440
\end{array}\right],
\end{aligned}
$$

$$
\begin{aligned}
& \boldsymbol{B}_{3}=\left[\begin{array}{cc}
-0.0049 & 0.0237 \\
0 & 0 \\
-4.0379 & 0.9613 \\
-0.0568 & -1.2168
\end{array}\right], \\
& \boldsymbol{B}_{4}=\left[\begin{array}{ccc}
-0.0069 & 0.0380 & -0.0153 \\
0 & 0 & 0 \\
23.3987 & 3.2993 & 21.4133 \\
-0.1644 & -1.9836 & 0.3313
\end{array}\right] .
\end{aligned}
$$

Der Zustandsvektor $\boldsymbol{x}_{i}=\left[\beta_{i} \Phi_{i} p_{i} r_{i}\right]^{\top}$ setzt sich aus dem Schiebe- und Rollwinkel sowie der Roll- und Gierrate zusammen. Als Eingangsgrößen dienen die Quer- und Seitenruderpositionen. Bei der F-18 kommt noch ein Stabilatorwinkel als dritte Eingangsgröße hinzu. Des Weiteren wurde bei der X-29 der geringfügige Einfluss der Aktoren auf den Schiebewinkel vernachlässigt. Der Kommunikationsgraph wird in Abbildung 1 dargestellt.

Das Ziel besteht darin die Schiebe- und Rollwinkel der Agenten zu synchronisieren. Dementsprechend lautet die Ausgangsmatrix

$$
C_{i}=\left[\begin{array}{llll}
1 & 0 & 0 & 0 \\
0 & 1 & 0 & 0
\end{array}\right], \quad \forall i \in\{1, \ldots, 4\} .
$$

Damit ergibt sich eine Differenzordnung von $\delta_{1,1}=2$ und $\delta_{2,1}=\delta_{3,1}=\delta_{4,1}=1$ bezüglich der ersten Ausgangsgröße und eine Differenzordnung von $\delta_{1,2}=\delta_{2,2}=\delta_{3,2}=\delta_{4,2}=2$ bezüglich der zweiten Ausgangsgröße. Als Synchronisierungstrajektorie wird für die Schiebewinkel eine Rampenfunktion und für die Rollwinkel ein sinusförmiges Signal angesetzt, sodass sich die Wunschpolynome zu $\bar{a}_{1}(s)=s^{2}$ und $\bar{a}_{2}(s)=s^{2}+1$ ergeben. Da für die Agenten 2, 3 und 4 die Differenzordnung bezüglich der ersten Ausgangsgröße geringer als die Ordnung des Wunschpolynoms ist, wird in diesem Übertragungspfad jeweils ein zusätzliches Verzögerungsglied der Ordnung 1 benötigt. Außerdem wird für die Rollwinkel eine exakte Trajektorienfolge angesetzt, wobei die Wunschtrajektorie durch

$$
\begin{aligned}
& \dot{\overline{\boldsymbol{x}}}_{2}=\left[\begin{array}{cc}
0 & 1 \\
-1 & 0
\end{array}\right] \overline{\boldsymbol{x}}_{2}, \\
& \bar{y}_{2}=\left[\begin{array}{ll}
2 & 1
\end{array}\right] \overline{\boldsymbol{x}}_{2},
\end{aligned}
$$

mit den Anfangswerten $\overline{\boldsymbol{x}}_{2}(0)=[0.040 .11]^{\top}$, explizit vorgegeben ist. Die zugehörige Folgeregelung wird für den Wurzelagenten 1 entworfen; alternativ hätte man auch den Wurzelagenten 3 wählen können. Für die Schiebewinkel wird keine explizite Wunschtrajektorie vorgegeben. Die Simulationsergebnisse sind in Abbildung 4 dargestellt. Es ist ersichtlich, dass die Synchronisierung nach ca. 4 Sekunden erreicht wird. Die Rollwinkel der Agenten synchroni- 

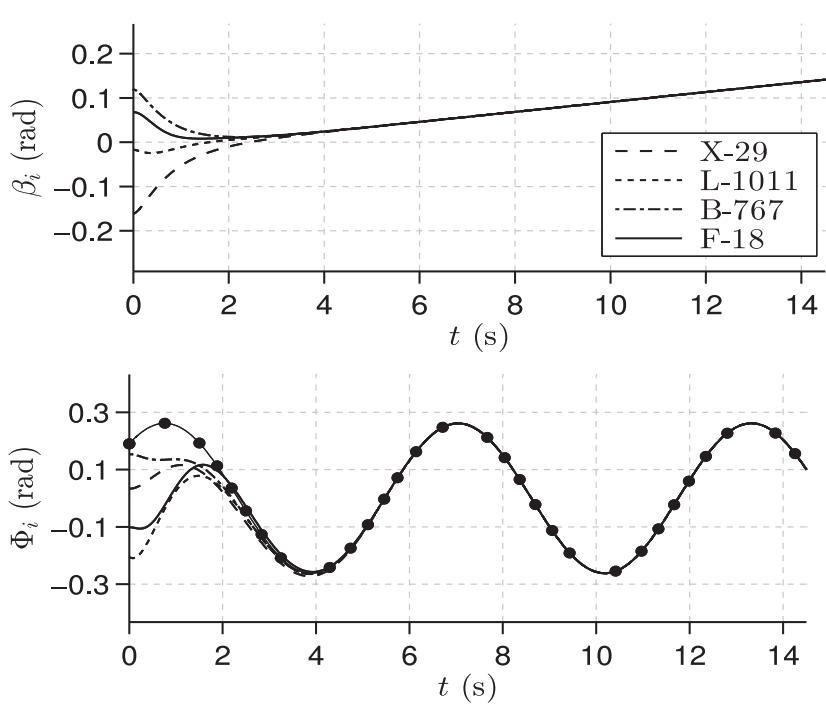

Abbildung 4: Synchronisierung der Schiebewinkel $\beta_{i}$ und Rollwinkel $\Phi_{i}$. Die gepunktete Linie im unteren Bild zeigt die explizit vorgegebene Wunschtrajektorie für die Rollwinkel.

sieren sich hierbei auf die vorgegebene Wunschtrajektorie (gepunktete Linie).

\section{Zusammenfassung}

In diesem Beitrag wurde eine Methode zur Synchronisierung heterogener linearer Multi-Agenten-Systeme präsentiert. Dabei wurde mit Hilfe des Entkopplungsreglerentwurfs eine Verfahrensweise vorgestellt, die es erlaubt die Ausgangsdynamik der Agenten zu homogenisieren und hierauf aufbauend bekannte Resultate für die Synchronisierung homogener Agenten anzuwenden. Dadurch lassen sich die Ausgangsgrößen nicht nur unabhängig voneinander beeinflussen, es kann auch eine explizite Dynamik vorgegeben werden. Darüber hinaus kann durch die einmalige Einführung eines autonomen Systems die Synchronisierungstrajektorie gezielt vorgeschrieben werden. Im Vergleich zu bestehenden Lösungsansätzen bietet die präsentierte Vorgehensweise eine einfache und effiziente Strategie, um heterogene Agenten zu synchronisieren und gleichzeitig die Dynamikordnung der Regler gering zu halten.

Danksagung: Diese Arbeit wurde durch die Deutsche Forschungsgemeinschaft (DFG) im Rahmen des GRK 1362 „Cooperative, Adaptive and Responsive Monitoring in Mixed Mode Environments“ (www.gkmm.de) gefördert.

\section{Literatur}

1. J. T. Bosworth. Linearized aerodynamic and control law models of the X-29A airplane and comparison with flight data. NASA Technical Memorandum 4356, 1992.

2. J. Descusse, J. F. Lafay, and M. Malabre. Solution to morgan's problem. IEEE Transactions on Automatic Control, 33(8):732$739,1988$.

3. C. Edwards and S. K. Spurgeon. On the development of discontinuous observers. International Journal of Control, 59(5):12111229, 1994.

4. P. L. Falb and W. Wolovich. Decoupling in the design and synthesis of multivariable control systems. IEEE Transactions on Automatic Control, 12(6):651-659, 1967.

5. J. A. Fax and R. M. Murray. Information flow and cooperative control of vehicle formations. IEEE Transactions on Automatic Control, 49(9):1465-1476, 2004.

6. O. Föllinger. Regelungstechnik: Einführung in die Methoden und ihre Anwendung. VDE Verlag, 2013.

7. T. I. Fossen. Mathematical models for control of aircraft and satellites, 2013. [Online] http://www.itk.ntnu.no/fag/gnc/ pensum.htm.

8. B. A. Francis. The linear multivariable regulator problem. SIAM Journal on Control and Optimization, 15(3):486-505, 1977.

9. C. Godsil and G. Royle. Algebraic Graph Theory. Springer, 2001.

10. H. F. Grip, T. Yang, A. Saberi, and A. A. Stoorvogel. Output synchronization for heterogeneous networks of non-introspective agents. Automatica, 48(10):2444-2453, 2012.

11. H. Kim, H. Shim, and J. H. Seo. Output consensus of heterogeneous uncertain linear multi-agent systems. IEEE Transactions on Automatic Control, 56(1):200-206, 2011.

12. G. Lafferriere, A. Williams, J. Caughman, and J. J. P. Veerman. Decentralized control of vehicle formations. Systems \& Control Letters, 54(9):899-910, 2005.

13. Z. Lin, B. A. Francis, and M. Maggiore. Necessary and sufficient graphical conditions for formation control of unicycles. IEEE Transactions on Automatic Control, 50(1):121-127, 2005.

14. K. D. Listmann. Synchronization of Networked Linear Systems - an LMI Approach. PhD thesis, Technische Universität Darmstadt, 2012.

15. K. D. Listmann, A. Wahrburg, J. Strubel, J. Adamy, and U. Konigorski. Partial-state synchronization of linear heterogeneous multi-agent systems. In Proceedings of the 50th IEEE Conference on Decision and Control and European Control Conference, pages 3440-3445, Orlando, 2011.

16. B. Lohmann. Vollständige Entkopplung durch dynamische Zustandsrückführung. at - Automatisierungstechnik, 39(9):459-464, 1991.

17. J. Lunze. Synchronization of heterogeneous agents. IEEE Transactions on Automatic Control, 57(11):2885-2890, 2012.

18. C.-Q. Ma and J.-F. Zhang. Necessary and sufficient conditions for consensusability of linear multi-agent systems. IEEE Transactions on Automatic Control, 55(5):1263-1268, 2010.

19. R. Olfati-Saber, J. A. Fax, and R. M. Murray. Consensus and cooperation in networked multi-agent systems. Proceedings of the IEEE, 95(1):215-233, 2007.

20. W. Ren. On consensus algorithms for double-integrator dynamics. IEEE Transactions on Automatic Control, 53(6):1503-1509, 2008. 
21. W. Ren, R. W. Beard, and E. M. Atkins. Information consensus in multivehicle cooperative control. IEEE Control Systems Magazine, 27(2):71-82, 2007.

22. G. Roppenecker and B. Lohmann. Vollständige Modale Synthese von Entkopplungsregelungen. at - Automatisierungstechnik, 36(11):434-441, 1988.

23. A. Saberi, A. A. Stoorvogel, and P. Sannuti. Control of Linear Systems with Regulation and Input Constraints. Springer, 2000.

24. J. M. Shewchun and E. Feron. High performance control with position and rate limited actuators. International Journal of Robust and Nonlinear Control, 9(10):617-630, 1999.

25. S. E. Tuna. LQR-based coupling gain for synchronization of linear systems, 2008. [Online] http://arxiv.org/abs/0801.3390.

26. A. Wahrburg and J. Adamy. Entkopplungsregelungen für lineare überaktuierte Systeme. at - Automatisierungstechnik, 61(1):28-38, 2013.

27. P. Wieland, R. Sepulchre, and F. Allgöwer. An internal model principle is necessary and sufficient for linear output synchronization. Automatica, 47(5):1068-1074, 2011.

28. C. W. Wu. Synchronization in networks of nonlinear dynamical systems coupled via a directed graph. Nonlinearity, 18(3):1057-1064, 2005.

29. T. Yang, A. Saberi, A. A. Stoorvogel, and H. F. Grip. Output synchronization for heterogeneous networks of introspective right-invertible agents. International Journal of Robust and Nonlinear Control, 2013. Zur Veröffentlichung angenommen.

\section{Autoreninformationen}

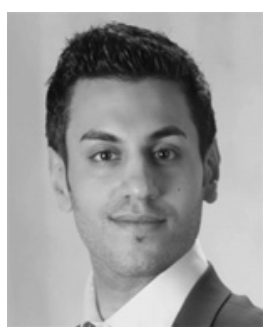

\author{
M.Sc. Saman Khodaverdian \\ Technische Universität Darmstadt, Institut \\ für Automatisierungstechnik, Fachgebiet \\ Regelungsmethoden und Robotik, \\ Landgraf-Georg-Str. 4, D-64283 Darmstadt, \\ Tel: +49-(0)6151-16-76053 \\ saman.khodaverdian@rmr.tu- \\ darmstadt.de
}

M.Sc. Saman Khodaverdian ist wissenschaftlicher Mitarbeiter am Fachgebiet Regelungsmethoden und Robotik im Institut für Automatisierungstechnik der Technischen Universität Darmstadt. Hauptarbeitsgebiete: Multi-Agenten-Systeme, verteilte und dezentrale Regelung, robuste Regelung.

Verfügbar unter

lediglich die vom Gesetz vorgesehenen Nutzungsrechte gemäß UrhG

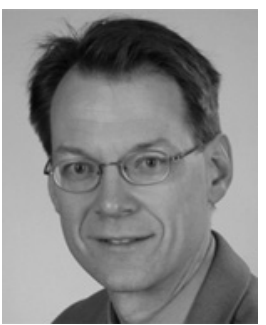

Prof. Dr.-Ing. Jürgen Adamy

Technische Universität Darmstadt, Institut für Automatisierungstechnik, Fachgebiet Regelungsmethoden und Robotik, Landgraf-Georg-Str. 4, D-64283 Darmstadt, Tel: +49-(0)6151-16-3442

jadamy@rmr.tu-darmstadt.de

Prof. Dr.-Ing. Jürgen Adamy ist Leiter des Fachgebiets Regelungsmethoden und Robotik im Institut für Automatisierungstechnik der Technischen Universität Darmstadt. Hauptarbeitsgebiete: Regelungsverfahren, Computational Intelligence, autonome mobile Roboter. 